\title{
Co-Development of an Infant Prototype in Hardware and Simulation based on CT Imaging Data
}

\author{
Benedikt Feldotto $^{1 *}$, Blasius Walch ${ }^{2}$, Patrick Koch ${ }^{1}$, Sebastian Dendorfer ${ }^{2}$, Alois Knoll ${ }^{1}$
}

\begin{abstract}
The development of biomimetic robots has gained research interest in the last years as it may both help understanding processes of motion execution in biological systems as well as developping a novel generation of intelligent and energy efficient robots. However, exact model generation that builds up on observations and robot design is very time intensive. In this paper we present a novel pipeline for co-development of biomimetic hardware and simulation models based on biological Computer Tomography (CT) data. For this purpose we exploit State of the Art rapid prototyping technologies such as 3D Printing and the Neurorobotics Platform for musculoskeletal simulations in virtual environments. The co-development integrates both advantages of virtual and physical experimental models and is expected to increase development speed of controllers that can be tested on the simulated counterpart before application to a printed robot model. We demonstrate the pipeline by generating a one year old infant model as a musculoskeletal simulation model and a print-in-place 3D printed skeleton as a single movable part. Even though we here only introduce the initial body generation and only a first test setup for a modular sensory and control framework, we can clearly spot advantages in terms of rapid model generation and highly biological related models. Engineering costs are reduced and models can be provided to a wide research community for controller testing in an early development phase.
\end{abstract}

\section{INTRODUCTION}

The human body is a highly complex biological system that is capable of sophisticated and coordinated but as well adaptive motion execution. These characteristics have gained research interest to benefit of understanding and reproducing the mechanics and control for robotic systems. The design of such biomimetic robot systems however is very time consuming as it consists of empirical experiments, data collection, modeling, design and testing. In this paper we introduce a novel pipeline that partially automates the procedure by building up on imaging technologies to generate a complete skeleton with functional joints in an iterative design approach. For this purpose we make use of CT imaging data, novel manufacturing technologies in particular 3D printing with print-in-place procedures and expect development speed-up by co-development of a virtual simulation model.

In this paper we describe the toolchain on the example of a one year infant model, an age that is of particular interest to understand the locomotion adaptation from crawling to

\footnotetext{
*Contact: feldotto@in.tum.de

${ }^{1}$ Robotics, Artificial Intelligence and Real-Time Systems, Faculty of Informatics, Technical University of Munich

${ }^{2}$ Laboratory of Biomechanics, Regensburg Center of Biomedical Engineering, Ostbayerische Technische Hochschule Regensburg
}

upright walking. According to the design principle of Embodiment [22] the imitation of natural body proportions and joint range of motions is of special interest as a properly designed body may already implement an important control aspect. Referring to the four anthropomimetic design principles introduced in [4] we here focus on body proportions, skeletal structure, and joint performance only. In simulation we demonstrate the manual adaptation of muscle arrangements with our introduced Robot Designer tool as major aspect of motion research.

\section{Biomimetic Robots And Musculos Keletal SIMULATIONS}

We introduce State of the Art in both biomimetic robot models as well as simulations for musculoskeletal robots. The Neurorobotics Platform, which is used for our experimental setup, is introduced as a general framework for biomimetic simulation and control.

\section{A. Technical Imitations of Human Musculoskeletal Systems}

Various approaches have been taken to build biomimetic humanoid robots which differ in the age that is modeled as well as in the principle and abstraction level in which the human musculoskeletal dynamics are imitated. Biological muscles are modeled with either tendon-driven, pneumatic or hydraulic technical implementations or purely in control algorithms. Popular adult robots with advanced motion capabilities can found in the compliantly controlled robot Justin (compliant control) [20], Kenshiro / Kengoro (tendondriven) [14], and a full scale musculoskeletal humanoid from the Suzumori Endo Lab at Tokyo Institut of Technology (multifilament pneumatic control) [15].

With particular interest in early learning of locomotion and human interactive learning several biomimetic infants are developed. The infant model "Pneuborn-13" [17] is equipped with 18 pneumatic McKibben actuators to study locomotion of crawling and walking. Roboy [25], as a follow up development of the ECCEROBOT [10] project, is a humanoid boy with $3 \mathrm{D}$ printed skeleton and 46 tendon actuators that mimic compliant control. The infant robot Noby [3] was developed at the Osaka University for interactive learning experiments. It is 71 centimeters tall and equipped with 600 tactile sensors, microphones and cameras. In [28] a musculoskeletal simulation model of a human foetus based on MRI and CT data is introduced for cortical learning experiments. 


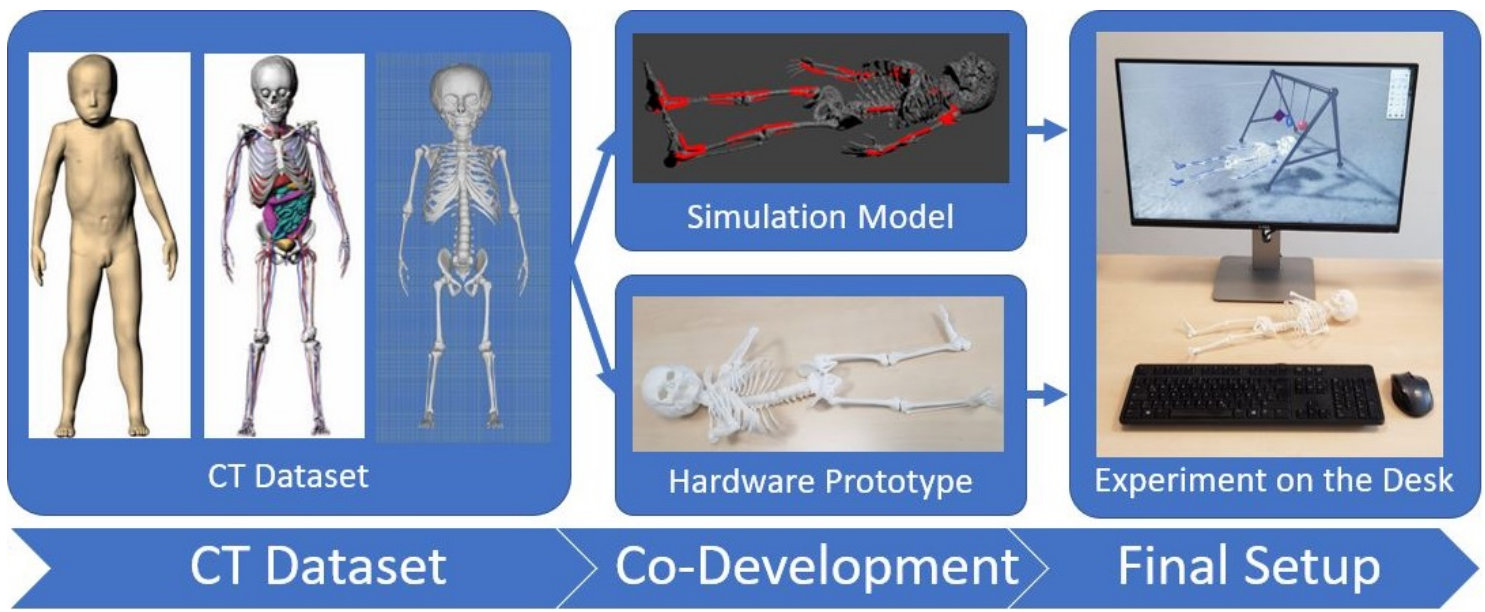

Fig. 1: Pipeline for Neurorobotic Model Generation: The diagram shows our proposed design pipeline for 3D printed biomimetic robots with high degree of biological realism. Skeleton data is retrieved from CT imaging data (Phantom images adapted from [18]), the final setup includes a simulation as well as a physical robot model. We introduce the Robot Designer as a graphical user interface to adapt simulation models, and technical model adaptation in CAD design. The working process is demonstrated on the example of a biomimetic infant but can be generalized for other musculoskeletal models as well.

\section{B. Simulation Tools for Musculoskeletal Bodies}

Despite customized simulations, frameworks for musculoskeletal simulations provide sophisticated analysis tools. Among the most popular ones, the OpenSim [26] and Anybody [6] simulators are developed to simulate musculoskeletal models for motion investigation and surgery planning. Here, the first one is offered open-source providing multiple interfaces, the latter one is optimized for the analysis of industrial designs. Several muscle types are supported based on experimental data. While OpenSim is integrated as part of the Neurorobotics Platform, AnyBody and its provided models were utilized to estimate the joint Center of Motions.

The Neurorobotics platforms [2] [1] is developed in scope of the Human Brain Project to enable embodied learning experiments in a virtual environment. It is an open-source and server-based simulation platform to connect both neural and body simulation in a comprehensive framework and hereby enables closed-loop simulations. The platform is build up on popular open-source toolboxes from robotics and neuroscience such as Gazebo [13], ROS [24] and NEST [9], respectively. The Neurorobotics Platform framework includes all tools necessary for experiment design and execution such as a Robot Designer, Environment Designer, State Machine and a Virtual Coach for scripted batch simulations.

\section{MethodS}

We select a suitable biological model of interest as an exemplary model for the modeling pipeline we introduce. As a baseline for the generated model we introduce the utilized CT dataset. Lastly, we introduce 3D printing technologies as the manufacturing procedure of interest.

\section{A. Selecting Human Age of Interest}

In Neurorobotics one major research questions is about how a complex multi-actuated musculoskeletal system imple- ments efficient but as well adaptive and robust control strategies for fine manipulation and locomotion. For humans these learning abilities evolve especially in the phase of learning to walk by starting with single uncoordinated motions towards crawling on the ground and finally getting upright. Empirical research spots the mean age for learning to crawl in the age of 31.26 weeks and for learning to walk alone after 58.14 weeks [8]. During this age the morphology of the skeleton supports this development by visible adaptation from a Cshape towards a S-shape spine [23]. We therefore select the reference age of about 52 weeks, a one year old infant, due to the experimental possibilities arising in this behavior learning period.

\section{B. Proposed Model Generation Pipeline}

We here introduce a model generation pipeline, that partially automates the modeling process but as well provides tools to tackle relevant research questions. Model generation is based on highly detailed CT data to ensure a desired degree of biologically realism, but as well simplify the modeling process by building up on existing geometric data. As visualized in Figure 1 we co-develop simulation and hardware prototype to speed up testing and implementation processes. For this purpose we introduce graphical model parametrization with our developed Robot Designer and CAD design, respectively. Finally, an experimental setup for robotics and neuroscience experiments on the desk is proposed that integrates advantages of both simulation and hardware model.

\section{Computed Tomography (CT) Dataset}

For investigation of human behavior learning and development of sensory-motor skills we base our model generation on highly detailed imaging data from real human models.

The data used in this thesis was generated at the Duke University in Northern Carolina by adapting CT data from 
a one-year-old participant to $3 \mathrm{D}$ surface data [18]. It has initially been generated for radio dosimetry prediction experiments and therefore contains detailed anatomies for skeleton, muscles and organs. From the dataset we depict the one year old male example, which has not yet undergone the S-spine transformation [23]. The infant data depicts the 50th percentile male selected to normal anatomy. The skeleton is taken from a patient which correlates well with the weight for age ratio, the modeled participant differs $3 \%(10.3 \mathrm{~kg} / 10$ $\mathrm{kg}$ ) in weight and $1.3 \%$ in height $(77 \mathrm{~cm} / 76 \mathrm{~cm})$ [18]. From the variety of imaged organs and structures in this first attempt we focus on the skeleton morphologies only.

\section{3D Printing Technology}

We exploit Rapid Prototyping technologies for short production cycles with minimal manufacturing efforts. Hereby we expect a speed up of iterative model testing and improvement. 3D Printing technologies induce several advantages for the production of our biomimetic skeleton model:

- complex geometry generation for bones/joints

- low cost production with off-the-shelf materials

- short cycle time in iterative design process

- simple reproduction by model file distribution

As the printing technology we choose Selective Laser Sintering (SLS) here on an EOS P110 printer. Advantages in contrast to Polyjet printing that speed up model generation or enable printing a humanoid skeleton are:

- No support material and surface treatment

- print-in-place including functional joints

- omni-directional uniform surface friction

- minimal layer size: 0.06-0.12mm

- bone imitation with spongy powder structures

Overall 3D printing enable fast model generation and ultimately also production at the customer place without expert knowledge.

Our model generation procedure is based on the experiments and findings in [5], which introduces the development of non-assembly 3D printed models and provides test series for proper parameter adjustment. We find support for the fact that SLS printing technologies are less fragile than Polyjet printed samples. Additionally, we extract proper joint designs with $0.3 \mathrm{~mm}$ clearance, a band cage ball joint design to reduce friction and easily remove excessive powder as well as a minimum joint radius of $5 \mathrm{~mm}$.

\section{Hardware Prototype}

We describe the technical adaptation of the biological skeleton towards a single part printable skeletal model. In particular, joints are substituted by basic technical implementations of revolute and ball/socket joints and the model is connected to a single part to retain experimental consistency. We also depict a proposed modular control architecture that we configured on a separate testbed.

\section{A. Skeleton}

While we keep the bone dimensions and arrangements, we substitute biological joints with technical implementations to ensure all time stable Center of Motion (COM) without the model falling apart. This adaptation also benefits from printin-place production without assembly to fasten production cycle times. For this initial model some simplifications are made to focus on main motion dynamics. Therefore, the hand and feet are modeled as single immobile parts and we only consider all joints with an accumulative Range of Motion (RoM) greater than 50. All joints are approximated as revolute and spherical technical joints. As an example in reference to [11] the shoulder and elbow joints are implemented as spherical and revolute joint, respectively. The lumbar spine is represented as two spherical joints between sacrum and the lumbar spine (Sacrum and L1) and between the lumbar spine and thoraric spine (L5 and TH12). The cervical spine is also represented as a spherical joint between two vertebraes of the cervical spine (C5 and C6). A test series established following parameters a clearance of $0.3 \mathrm{~mm}$ and specific dimensions for spherical joints (band width $3 \mathrm{~mm}$, radius $5.5 \mathrm{~mm}$ ) and revolute joints (radius $3.5 \mathrm{~mm}$ ).

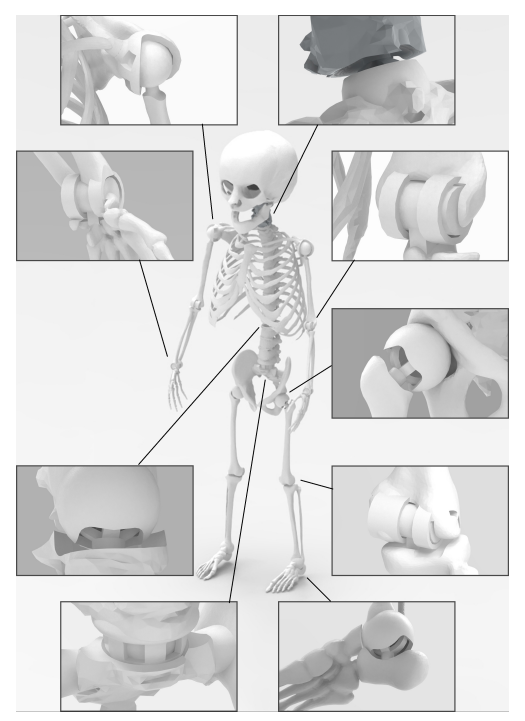

Fig. 2: CAD design of the infant prototype: The biological skeleton joints are substituted with technical imitations, preserving center and range of motion. Minimal changes retain maximal biological realism, while exploiting 3D printing capabilities. The skeleton can be printed in place with functional joints, no post assembly steps needed.

The joint design process is executed in three adaptation steps: (1) Specify Center of Rotation, (2) Implement a suitable technical joint type [revolute, ball and socket], (3) Adapt range of motion by technical constraints. Figure 2 shows the overall adapted model and visualizes the implemented joint types in closeups. To even further minimize costs we printed the skeleton model in scale 1:2. In total we adapted 15 joints with a total of 33 Degrees of Freedom.

The overall skeletal and single bone dimensions are nat- 


\begin{tabular}{lcccc}
\hline Location & $\begin{array}{c}\text { Joints } \\
\text { Ball \& Socket }\end{array}$ & Revolute & & DoF \\
\hline Lumbar spine & $\mathrm{x}$ & & 2 & 3 \\
Cervical Spine & $\mathrm{x}$ & & 1 & 3 \\
Shoulder & $\mathrm{x}$ & & 2 & 3 \\
Elbow & & $\mathrm{x}$ & 2 & 1 \\
Wrist & $\mathrm{x}$ & $\mathrm{x}$ & 2 & 1 \\
Hip & & $\mathrm{x}$ & 2 & 3 \\
Knee & $\mathrm{x}$ & & 2 & 3 \\
Ankle & 9 & 6 & 15 & 33 \\
\hline Total & & & &
\end{tabular}

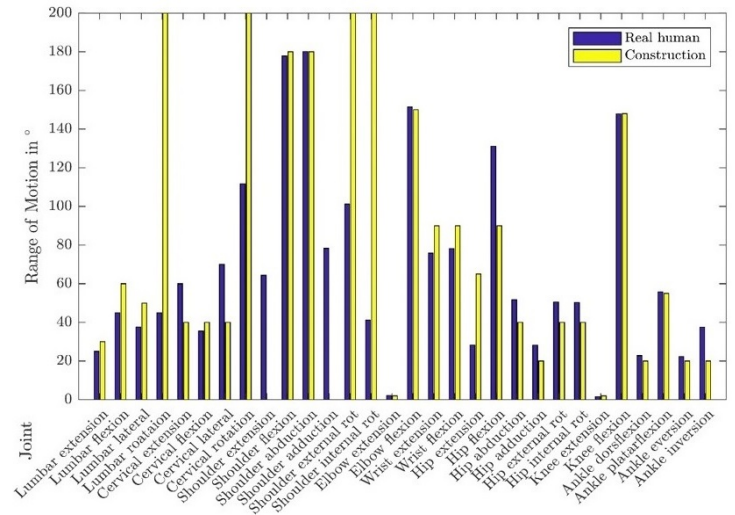

Fig. 3: Infant Prototype Joints: Implemented joints and total Degree of Freedom (left), comparison of joint range of motions of our prototype to empirical data of human joints (right)

urally evaluated as they are based on the CT imaging data. Since no complete empirical dataset for all joint Range of Motions exist for a one year old infant [21], we amalgamate data for the lumbar spine [12], the cervical spine [16][19], hip, knee, ankle, shoulder, elbow and wrist[27][21]. As a backup for some joints data of eight year old participants or adult data was used, both are expected in the actual infant range $+-10^{\circ}[27]$.

Figure 3 lists the individual and total sum of the implemented Degrees of Freedom. On the right side a direct comparison for the designed joint Range of Motions extracted from CAD design in contrast to the biological empirically derived ones is made. The shoulder adduction and the shoulder extension was not implemented in the model thus the values are 0 . We could map most joints with difference of about $+-30^{\circ}$ or better. The chosen spherical design type did not include rotational limits, therefore range of rotation for all ball and socket joints is left infinite.

The production accuracy of the 3D printer varied and led to some joints stuck with powder and hereby left immovable in the printed model. Pre-tests evaluated a clearance $>0.5$ $\mathrm{mm}$ enough including all production variances. We therefore recommend printing the model in original size instead of downscaled which would leave enough space bigger than the $0.3 \mathrm{~mm}$ we used to ensure reliable and unrestricted movability considering production tolerances.

For a generic femoral bone exemplified as a cylindric structure we tested the advantages replicating a spongy structure found in the mammalian skeleton. We design a hollow cylinder in one of the bones. As the raw powder material cannot escape from within this structure the SLS printing technology will hereby create a rather spongy inner material structure. Figure 4 shows results of a non-standardized 3point bending test with solid and spongy bone structure. We explore an increase of flexibility for the latter one, which may be beneficial for efficient motion execution by adaptation to environmental constraints. As differences occur only at forces greater than 90 Newton, the structure is not relevant for our small body model but may have an impact on future bigger model structures.
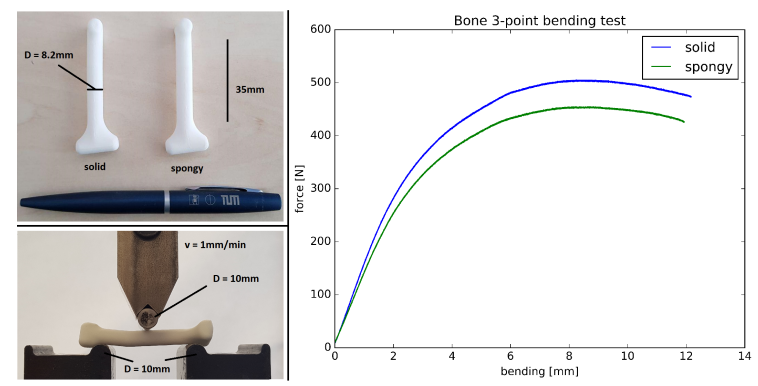

Fig. 4: Using Selective Laser Sintering (SLS) 3D printing technologies we can partially imitate biological bone characteristics. Here we compare a solid to rather spongy bone structure in a 3-point bending test (setup left bottom). Differences occur with forces $>90 \mathrm{~N}$, maximal forces are 504 and $354 \mathrm{~N}$ for the solid and spongy material, respectively.

\section{Control and Sensor Modalities}

From the five human senses Audition, Vision, Tactile, Olfaction and Taste the first three are most interesting for human interaction and learning of motion coordination and shall be implemented in the infant model. For best usability as a test setup for neural learning algorithms we aim a modular approach, any controller implementation has full access to all sensed information. In a test setup we exploit ROS as a communication layer which runs on a Raspberry Pi Zero that fits into the infants chest. The initially poor sensor capabilities of infantile biological creatures justify low cost hardware solutions that may include sensory inaccuracies. We demonstrate potential implementations of sensory feedback with a Marviotek Endoscope (CMOS, view angle $66^{\circ}$, resolution 720p) and Adafruit MEMS (range of $100 \mathrm{~Hz}$ - $10 \mathrm{KHz}$ ) for vision and audition, respectively, and use common libraries for biomimetic data processing. Hereby we can implement edge detection as well as primitive object detection that mimic very basic principles of lower level visual neural circuits with OpenCV tools, audio input is processed with a Fast Fourier Transform (FFT) to provide frequency feedback according to the human cochlea. The control architecture was tested on a separate rack not visual- 
ized in the pictures, the control board fits in the infant chest, the cameras into the eye holes and will be integrated soon. Communication to e.g. the Neurorobotics Platform can be established via ROS and on board WLAN communication.

\section{Simulation}

The co-development of a digital twin in simulation is expected to speed up iterative development cycles. Several advantages include no suffering from wear and tear, model multiplication without cost and the ability to control with unpredictable output such as from Artificial Neural Networks. The skeleton model is simulated as a rigid body simulation with the SimBody physics engine including additional application of muscle forces. To build musculoskeletal simulation models the Robot Designer [7] as a plugin in the 3D modeling suite blender is implemented.

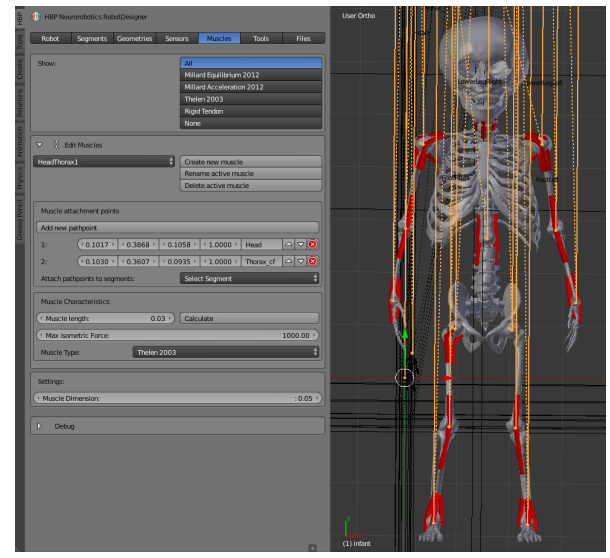

Fig. 5: Model Preparation in our Robot Designer as a Plugin for Blender: With a graphical user interface we can adapt the $3 \mathrm{D}$ robot model, change kinematic and dynamic parameters. As a particular aspect of motion (locomotion, manipulation etc.) research muscle attachment and routing points can be graphically defined. The exported package can directly be used to simulate in the Neurorobotics Platform.

Figure 5 shows the graphical user interface to adapt dynamics and kinematics parameters. A special interest in motion research is the actuation by contractile and flexible muscles. Within the Robot Designer muscle attachment points can easily be drawn into the model for rapid testing of different configurations. A selection of various muscle types with different characteristics is provided, that also include technical imitations of biological characteristics. In simulation we can build up on a rich modular library for sensor modalities provided as Gazebo ROS plugins.

\section{Neurorobotic EXPERIMENTS ON THE Desk}

Both simulation and hardware prototype model can be used for experiments on the intersection of robotics and neuroscience. Figure 6 conceptually shows the proposed test setup on the desk. Since both simulation and hardware model are based on ROS communication protocols, both can easily be substituted, or in general controller tested in simulation transferred to the physical model for parameter finetuning and interactive experiments. In simulation we can control the infant model by means of spiking or classical neural networks tested in closed-loop simulations. The hardware model may be used for morphological motion studies for now and once actuation and sensation is integrated for human interactive learning experiments.

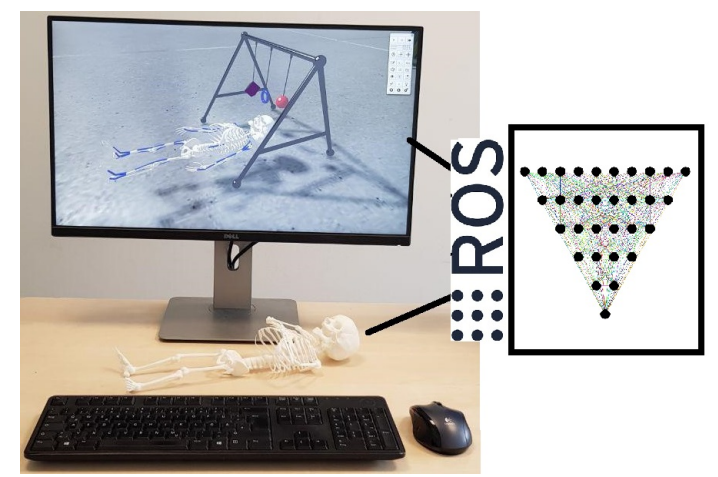

Fig. 6: Conceptual drawing of our proposed experiment setup on the desk: Based on CT imaging data we co-design a simulation and hardware model of a one year old infant. Simulation in the Neurorobotics Platform enables neural learning experiments on a musculoskeletal model, providing a benchmark experiment in simulation. The 3D printed skeleton will be equipped with vision and auditory sensors we tested separately, as a basis for interactive experiments. Both control structures are based on ROS which enables a fluent switch to apply control algorithms developed in simulation on the hardware model.

\section{CONCLUSION}

As a result we can demonstrate a $3 \mathrm{D}$ printed infant skeleton as a single part with functional joints. In the Neurorobotics Platform we can simulate the same model and add muscle dynamics for simulation of motion patterns. We also depict a first approach for a sensory control architecture for audition and vision that is modular based on ROS. This paper describes the proposed pipeline and implemented tools as well as technologies with focus on the skeleton in hardware and muscle actuation in simulation. We can demonstrate a fast development process from imaging data to a full model that is low-cost, modular, scalabe and biomimetic. The introduced pipeline is general in a way that it can be transfered to modelling of other mammalian bodies.

While robots with higher capabilities exist, here we present the first print-in-place robot infant model. Building up on CT imaging data as well as co-development of hardware and simulation model from the start can be considered as novel in our approach. The benefits are obvious in terms of low cost, fast prototyping, biological realism as well as modular control. The approach described in this paper is limited as an initial attempt, the hardware prototype only considers a skeletal model and a test bed for control that will be integrated in the infant body soon. After printing we found several joints stuck due to manufacturing inaccuracies, printing the model in original size is expected to help out here 
and may also reduce fragibility of the overall model. We only modeled basic joint types such as revolute and ball/socket joints in both hardware and simulation, only joints with motion range greater than 50 degree have been implemented. The simulation is limited to rigid body simulation with applied muscle forces, collision geometries need to be defined for environment interaction experiments. Joint motion ranges have been evaluated in comparison to constituted empirical biological validation datasets as no global dataset yet exits for a one year infant.

Future work include a refinement of the model, which may include evaluation of muscle attachment points with the CT data set and implementation of sensors and actuators for both simulation and hardware prototype model. Printing the model without downscaling is expected to tackle discovered issues in robustness and reliability of the hardware model. The dataset contains models of different ages (newborn, 5, 10, 15 years) which can be modeled with our proposed pipeline as well, but also other biological mammals may be replicated in hardware in simulation with our approach. The gained knowledge then may be used to even further optimize the models technically for a new generation of bio-inspired robots. Our pipeline introduces the generation of a digital or $3 \mathrm{D}$ printed twin of human participants that may may help doctors to better understand and treat personalized motion disabilities in the future.

\section{ACKNOWLEDGMENT}

This research has received funding from the European Unions Horizon 2020 Framework Programme for Research and Innovation under the Specific Grant Agreement 720270 (HBP SGA1) and No. 785907 (Human Brain Project SGA2). We like to thank Prof. Dr. W. Paul Segars, Duke University Medical Center, for providing the infant skeleton data described in [18]. We like to thank Luciano Avila Gray and Daniel Amrein (both TUM) for supporting the bending test.

\section{REFERENCES}

[1] Ugo Albanese et al. Hbp Neurorobotics Platform. 2018. DoI: 10 . 5281/ZENODO.1296203.

[2] Alois Knoll and Marc-Oliver Gewaltig. "Neurorobotics: A strategic pillar of the Human Brain Project". In: Science/AAAS, (2016), pp. $25-34$.

[3] AP Archive. Toddler-bot Noby to help shed light on how humans learn. 2015. URL: https://www. youtube.com/watch?v= NCnfIDSo $7 \mathrm{ks}$.

[4] Yuki Asano, Kei Okada, and Masayuki Inaba. "Design principles of a human mimetic humanoid: Humanoid platform to study human intelligence and internal body system". In: Science Robotics 2(13):eaaq0899 (). DOI: $10.1126 /$ scirobotics . aaq0899..

[5] Jacques Cali et al. "3D printing of non-assembly, articulated models". In: ACM Transactions on Graphic 2012 (31(6):1). DOI: 10 . $1145 / 2366145.2366149$.

[6] Michael Damsgaard et al. "Analysis of musculoskeletal systems in the AnyBody Modeling System". In: Simulation Modelling Practice and Theory 14.8 (2006), pp. 1100-1111. ISSN: 1569190X. DOI: 10.1016/j.simpat.2006.09.001.

[7] Benedikt Feldotto, Guang Chen, and Stefan Ullbrich. The BlenderRobotDesigner of the Neurorobotics Platform. Munich. URL: https : / / github . com / HBPNeurorobotics / BlenderRobotDesigner / tree / master (visited on 05/10/2019).
[8] M. Garret. "Locomotor milestones and babywalkers: Cross sectional study." In: BMJ 324(7352):1494 (2002). DOI: $10.1136 / \mathrm{bmj}$. 324.7352 .1494 .

[9] Marc-Oliver Gewaltig and Markus Diesmann. "NEST (NEural Simulation Tool)". In: Scholarpedia 2.4 (2007), p. 1430. DOI: 10 . 4249/scholarpedia.1430.

[10] Hugo Gravato Marques et al. "ECC E1: $t$ he first of a ser ies of anthropomimetic musculoskeletal upp ertorsos". In: IEEE-RAS International Conference on Humanoid Robots (2010). URL: https: / / ieeexplore. ieee. org / stamp / stamp. jsp ? tp = \&arnumber $=5686344$.

[11] M. Jäntsch et al. "Anthrob - A printed anthropomimetic robot". In: 2013 13th IEEE-RAS International Conference on Humanoid Robots (Humanoids). 2013, pp. 342-347. DOI: 10 . $1109 /$ HUMANOIDS . 2013.7029997.

[12] G. K. Fitzgerald et al. "Objective assessment with establishment of normal values for lumbar spinal range of motion". In: Physical therapy 63(11):1776-1781 (1983).

[13] N. Koenig and A. Howard. "Design and use paradigms for gazebo, an open-source multi-robot simulator". In: 2004 IEEE. IEEE, 2004 pp. 2149-2154. ISBN: 0-7803-8463-6. DOI: 10.1109 / IROS . 2004.1389727.

[14] Toyotaka Kozuki et al. "Muscle-tendon complex control by "Tension controlled Muscle" and "Non-linear Spring Ligament" for real world musculoskeletal body simulator Kenshiro". In: (2014). URL: http://ieeexplore.ieee.org/document/6913891/.

[15] S Kurumaya et al. "Musculoskeletal lowerlimb robot driven by multifilament muscles". In: Robomech Journal 3.18 (2016).

[16] Bengt Lind et al. "Normal range of motion of the cervical spine". In: Archives of Physical Medicine and Rehabilitation 70.9 (1989), pp. 692-695. DOI: 10.5555/uri:pii:

[17] K Narioka et al. "Pneumatic musculoskeletal infant robots". In: IEEE ICRA (2009).

[18] Hannah Norris et al. "A set of 4D pediatric XCAT reference phantoms for multimodality research." In: Medical physics 41(3):033701 (2014.). DOI: $10.1118 / 1.4864238$. URL: https : / / aapm . onlinelibrary.wiley.com/doi/abs / 10.1118 / 1 . 4864238.

[19] Anna Maria Ohman and Eva R. E. Beckung. "Reference values for range of motion and muscle function of the neck in infants." In: Pediatric physical therapy: the official 20.1 (2008), pp. 53-58. DOI: 10.1097/PEP.0b013e31815ebb27.

[20] Ch. Ott et al. "A Humanoid Two-Arm System forDexterous Manipulation". In: 6th IEEE-RAS International Conference on Humanoid Robots (2006).

[21] Anne Parrot, Michale Tousignant, and Yvan St-Cyr. Normative Reference Values for Musculoskeletal Conditions Normative Reference Values for Musculoskeletal Conditions and Functional Motor Abilities in the Pediatric Population - Literature Review and Clinical Guidlines. Quebec, 2010. ISBN: 978-2-922242-44-7.

[22] Rolf Pfeiffer and Josh Bongard. How the Body Shapes the Way We Think: A New View of Intelligence. 2007. ISBN: 978-0262162395.

[23] Jan P. Piek. Infant motor development. Human Kinetics. Champaign, IL, 2006. ISBN: 073600226X.

[24] Morgan Quigley et al., eds. ROS: an open-source Robot Operating System. 1/2009.

[25] Rolf Pfeifer, Hugo Gravato Marques, Fumiya Iida. "Soft Robotics: The Next Generation of Intelligent Machines". In: 23rd International Joint Conference on Artificial Intelligenc (International Joint Conference on Artificial Intelligenc).

[26] Ajay Seth et al. "OpenSim: Simulating musculoskeletal dynamics and neuromuscular control to study human and animal movement". In: PLoS computational biology 14.7 (2018), e1006223. DOI: 10 . 1371/journal.pcbi.1006223.

[27] J. M. Soucie et al. "Range of motion measurements: Reference values and a database for comparison studies". In: Haemophilia : the official journal of the World Federation 17.3 (2011), pp. 500-507. DOI: $10.1111 / j .1365-2516.2010 .02399 . x$.

[28] Yasunori Yamada et al. "Am Embodied Brain Model of the Human Foetus". In: 6.27893 (2016). DOI: 10.1038 / srep27893. URL https://www. nature.com/articles/srep27893.pdf. 